Revista peruana de biología 26(2): 283 - 288 (2019) doi: http://dx.doi.org/10.15381/rpb.v26i2.16382 ISSN-L 1561-0837; elSSN: 1727-9933

Universidad Nacional Mayor de San Marcos

\section{Sobre la importancia de la certificación internacional para los anilladores de aves en Perú}

\author{
About international certification importance for Bird Banders \\ in Peru
}

$\begin{array}{ll}\text { Presentado: } & 12 / 12 / 2018 \\ \text { Aceptado: } & 04 / 03 / 2019 \\ \text { Publicado online: } & \text { 06/07/2019 }\end{array}$

\section{Correspondencia:}

*Autor para correspondencia

Alexis Díaz: alexis.diaz@corbidi.org

Flor Hernández: fhernandez@corbidi.org

Mauricio Ugarte: mugartelewis@gmail.com

Julio Salvador: julio.salvador.r@gmail.com

Eveling Tavera: etavera@corbidi.org

Diego García: dgolae@corbidi.org

Rachel Kilby: rachel_kilby@hotmail.com

Thomas Valqui: tvalqui@corbidi.org

Otros datos de los autores / biografía:

ORCID Alexis Díaz: 0000-0003-3291-562X

ORCID Flor Hernández: 0000-0002-6709-4764

ORCID Mauricio Ugarte: 0000-0002-1571-4263

ORCID Julio Salvador: 0000-0001-9501-3346

ORCID Eveling Tavera: 0000-0003-0058-6569

ORCID Rachel Kilby: 0000-0003-0399-7902

\section{Citación:}

Díaz A., F. Hernández, M. Ugarte, J. Salvador, E. A. Tavera, D. García, R. Kilby, T. Valqui. 2019. Sobre la importancia de la certificación internacional para los anilladores de aves en Perú. Revista peruana de biología 26(2): 283 - 288 (Mayo 2019). doi: http:// dx.doi.org/10.15381/rpb.v26i2.16382

Palabras clave: certificación; anillamiento de aves; anillador; Consejo Norteamericano de Anillado; Perú

Keywords: certification; bird banding; bander; North American Banding Council; Peru.

\section{Alexis Díaz*1,2,3, Flor Hernández $1,2,4$, Mauricio Ugarte ${ }^{1,5}$, Julio Sal- vador $^{1,2,6}$, Eveling A. Tavera ${ }^{1,2,7}$, Diego García ${ }^{1,2,8}$, Rachel Kilby ${ }^{1,9}$, Thomas Valqui* 2}

1. North American Banding Council (NABC), 13551 W State Route 2, Oak Harbor, OH 43449. USA

2. Centro de Ornitología y Biodiversidad (CORBIDI), Santa Rita No 105, Dpto. 202, Urb. Huertos de San Antonio, Surco Lima, Perú

3. Department of Biology, University of Miami, Coral Gables, FL 33146, USA.

4. Department of Biological Sciences, University of Texas El Paso, El Paso, TX 79968, USA

5. Universidad Nacional de San Agustín de Arequipa, Museo de Historia Natural (MUSA), Colección Científica, Área de Ornitología. Av. Alcides Carrión s/n, Arequipa, Perú.

6. Universidad Nacional Agraria La Molina. Av. La Molina S/N. Lima - Perú.

7. Centre for Wildlife Ecology, Department of Biological Sciences, Simon Fraser University, 8888 University Drive, Burnaby, British Columbia V5A 1S6, Canada.

8. Department of Wildlife Ecology and Conservation. University of Florida. Gainesville, FL 32611-0430, USA.

9. Reserva Ecológica Taricaya, Madre de Dios, Perú.

\section{Resumen}

La certificación como anillador de aves consiste en validar el nivel de conocimiento y experiencia de personas frente a un estándar consolidado de trabajo en el ejercicio de la captura, anillado, manejo, y liberación de aves, con el fin de promover la seguridad del ave, la seguridad del anillador y la calidad de los datos colectados. El Consejo Norteamericano de Anillado (NABC) es una organización sin fines de lucro, engloba a diversas organizaciones de investigación, conservación y gestión de la avifauna en Norteamérica. Continuando con su principal misión de promover prácticas responsables de la técnica del anillado de aves bajo criterios estandarizados y éticos, en un trabajo conjunto con el Centro de Ornitología y Biodiversidad (CORBIDI) y otras instituciones afines, entre el 2017 y el 2018 se han desarrollado las tres primeras sesiones de certificación NABC en el Perú. El presente trabajo intenta responder algunas de las preguntas más frecuentes relacionados con estas sesiones de certificación, así como los esfuerzos del anillado de aves promovidos por el Programa de Anillamiento CORBIDI (PAC).

Abstract

Certification as bird bander implies to validate the level of knowledge and experience of people with regard to a consolidated standard of work in the exercise of capture, banding, handling, and release of birds, in order to promote the safety of the bird, the security of the bander and the quality of the data collected. The North American Banding Council (NABC) is a non-profit organization that includes diverse organizations of research, conservation and management of North American avifauna. Continuing with its mission to promote responsible bird-banding practices under standardized and ethical criteria, in a joint effort with the Centro de Ornitologia y Biodiversidad (CORBIDI) and other related institutions, the first three NABC certification sessions were recently held in Peru. This work tries to answer some of the most frequent questions related to these certification events and the bird banding efforts promoted by the Programa de Anillamiento CORBIDI (PAC). 


\section{Introducción}

La certificación y el entrenamiento constante de profesionales y aficionados dedicados al anillamiento de aves promueve eficiencia y estandarización en la captura, el manejo y el bienestar de las aves, así como en la calidad de la colecta, análisis e interpretación de datos sobre la biología de éstas (Doscher 2005, NABC 2003a). El anillamiento de aves con fines científicos se remonta a más de 100 años con los esfuerzos del danés Hans Christian Cornelius Mortensen en su afán por estudiar la migración de las aves (Baillie et al. 2009). Desde ese entonces, esta técnica, que consiste en la individualización de las aves mediante el uso de anillos metálicos con un código único (Villarán 2002) se ha convertido en una herramienta de gran utilidad para estudios sobre migración, dinámica poblacional, comportamiento, muda, condición corporal, productividad y supervivencia, ecología de comunidades, y conservación en diversas regiones del mundo (Herman 1944, Ralph et al. 1996, Buckley et al. 1998, Pinilla 2000, Guallar 2015).

El uso del anillamiento científico en programas de monitoreo de poblaciones de aves silvestres se ha desarrollado y usado ampliamente en el hemisferio norte (especialmente en Europa y Norteamérica), en donde la práctica de esta técnica requiere de un permiso de anillado otorgado a personas con probada instrucción y experiencia (de aquí en adelante "anillador") (Tautin 2005, Baillie et al. 2007). Las instituciones oficiales encargadas de emitir estos permisos son por lo general agencias gubernamentales o instituciones de investigación, como la Unión Europea para el Anillamiento de Aves (EURING), el Fondo Británico para la Ornitología (BTO), el Servicio Geológico de los Estados Unidos (USGS) y el Servicio de Vida Silvestre de Canadá (CWS). En el Perú, el anillamiento de aves ha sido aplicado en ámbitos académicos y planes de manejo para la conservación de especies de importancia económica desarrollados por cortos periodos de tiempo (Franke, 2012). Dichos trabajos han involucrado el estudio de diversos grupos taxonómicos tales como aves marinas (Vogt 1942, Koepcke 1959, Jordán \& Cabrera 1960, Fuentes 1971, Zavalaga \& Paredes 1997, Calvo 2016), playeras (Myers et al. 1990, Villavisencio 1990, Sarango 1992, Pulido et al. 1996, Tavera et al. 2016), paseriformes (Gonzáles \& Málaga 1997, Gonzáles \& La Torre-Cuadros 2001, Gonzales 2004, Nolazco \& Roper 2014, UngvariMartin et al. 2016, Scholer et al. 2018, Díaz et al. 2018, Chumpitaz-Trujillo et al. 2018, Kennedy et al. 2018, Torres-Vásquez et al. 2018) y rapaces (Beingolea 2009).

En el 2009, se realizó el primer curso internacional de anillamiento de aves en Perú, organizado por el Consejo Norteamericano de Anillado (NABC), la Red de Anillamiento de Aves del Hemisferio Occidental (WHBBN) y la Estación de anillamiento Taricaya. Posteriormente, en el 2010 y durante el desarrollo de la cuarta conferencia del WHBBN en Brasil, se anunció la creación del Programa de Anillamiento CORBIDI (PAC) (Moreno et al. 2011). En asociación con Porzana Ltd. y The Wetland Trust y con el financiamiento del Acta para la Conservación de las Aves Migratorias Neotropicales del Servicio de Pesca y Vida
Silvestre de los Estados Unidos (NMBCA-USFWS), CORBIDI logró obtener anillos y equipo que permitieron impulsar diversas investigaciones usando el anillamiento como herramienta para el monitoreo de aves silvestres en el Perú (Nolazco \& Roper 2014, Díaz et al. 2015, Tavera et al. 2016, Díaz \& Dimming 2017, Alza et al. 2017, Guevara-Torres et al. 2017, Díaz et al. 2018). Se han realizado, además, simposios, disertación de tesis (Díaz 2015, Mandujano-Collantes 2016, Chumpitaz-Trujillo 2018, Antezana 2019), talleres y cursos de anillado, con el fin de promover el uso y resaltar la importancia del anillamiento para el estudio de aves silvestres. Sin embargo, a pesar de los esfuerzos puntuales antes mencionados, todavía se está lejos de tener un programa nacional coordinado para anillamiento que aporte al conocimiento de la gran diversidad de aves que existen en el Perú. De esta manera, se considera que se necesita aún más anilladores calificados de aves en el país, para hacer más extensiva esta iniciativa y permita consolidarse en el largo plazo. Más aún, no hay una normatividad de la técnica de anillado a nivel nacional, la que podría lograrse a través de un proceso de certificación reconocida en medios académicos. En ese sentido, el NABC ha impulsado la realización de sesiones de certificación profesional en Latinoamérica y el Caribe, siendo tres de estas recientemente realizadas en el Perú.

\section{¿Por qué es importante estar certificado como anillador de aves?}

Optar por obtener una certificación como anillador significa validar el nivel de conocimientos y experiencia de personas frente a un estándar consolidado de trabajo en el ejercicio del anillamiento de aves. Una certificación genera confianza, tanto para el anillador como para aquellas instituciones que requieren un sistema para calificar a una posición de trabajo en el anillado de aves. La certificación además permite a las organizaciones de anillado, tales como los observatorios de aves, conseguir y mantener criterios estandarizados de trabajo y realizar un seguimiento de la técnica de anillado como herramienta en el estudio de la biología de las aves. Esto a su vez, promueve el nivel de aceptación de estos proyectos por parte de la comunidad científica, así como el público en general.

¿Qué es el Consejo Norteamericano de Anillado y cuál es su misión?

El Consejo Norteamericano de Anillado (NABC) es una organización sin fines de lucro conformado por representantes de diversas organizaciones involucradas en la investigación, conservación y gestión de la avifauna de Estados Unidos y Canadá, contando también con la presencia de entidades en América Latina, tales como Brasil, Costa Rica y México. Su principal misión es promover las prácticas responsables de la técnica de anillado de aves bajo criterios estandarizados y éticos (NABC 2003a). Este consejo ha desarrollado un programa de entrenamiento y certificación a fin de establecer estándares de conocimiento y experiencia en tres niveles: Asistente, Anillador e Instructor (NABC 2003b). La certificación, además, es otorgada con mención a cinco 
diferentes grupos taxonómicos de aves: paseriformes y cuasipaseriformes, rapaces, playeras, anátidos y colibríes (NABC 2003a). Mediante el entrenamiento y certificación de anilladores, el NABC busca fomentar la seguridad del anillador, la seguridad del ave y la calidad de los datos de investigación obtenidos.

¿Por qué optar por una certificación y estándares promovidos por el NABC en Sudamérica?

Actualmente no existe institución alguna que evalúe y certifique los niveles de competencia de anilladores trabajando en el Perú. La institución de anillado más cercana a nuestro país y que cuenta con un programa de entrenamiento y certificación consolidado, a nivel nacional e internacional, es el NABC. Institución que, hasta finales del 2018, ha logrado certificar a 13 profesionales peruanos bajo sus estándares. Esto se logró a través de pasantías en diversos observatorios de aves, tales como el Observatorio de aves de Klamath (KBO) y el Observatorio de aves de Long Point (LPBO), y sesiones de certificación celebrados en reuniones del Grupo de Aves Playeras del Hemisferio Occidental (WHSG) en Norteamérica. Esto manifiesta el interés de estas instituciones por afianzar los estándares NABC en el programa de anillado del Perú, ya que aproximadamente el $56 \%$ de las especies de aves neárticas migran hacia sus territorios de invernada en el Neotrópico (Stotz et. al. 1996). Por tal motivo, un trabajo estandarizado por parte de diversos programas de anillamiento a lo largo del continente americano resulta importante con el fin de estudiar la dinámica de sus poblaciones de manera efectiva.

\section{¿Qué especialización existe en los diversos nive-} les de certificación NABC?

El primer nivel de certificación es el de 1) Asistente, en el que el estudiante ha obtenido las competencias básicas en las técnicas de captura de aves, así como la manipulación y anillado de aves bajo la supervisión de un Anillador o Instructor. 2) Anillador, en el que el estudiante ha alcanzado un nivel de competencia en la captura, identificación, manipulación y anillado de aves, determinación de edad y sexo, toma de medidas, y el adecuado manejo y mantenimiento de datos de anillado suficientes para operar una estación de anillado sin una supervisión directa (NABC 2003a). Finalmente, 3) Instructor, con un nivel de exigencia superior a los anteriores. Un instructor certificado deberá no solo manejar las competencias básicas del anillado de aves, sino también demostrar sus habilidades de enseñanza y criterio para evaluar futuros aspirantes a la certificación (NABC 2003b). Además, el Instructor queda facultado para certificar a otros anilladores en futuras sesiones de certificación a nivel nacional o internacional.

\section{¿Hay suficientes anilladores certificados en el} Perú?

A pesar de que el Perú es el tercer país con mayor diversidad de aves en el mundo (Remsen et al. 2018) y el número de estudios ornitológicos relacionados con la historia natural de las aves en el país se han incrementado (Plenge 2018), aún existen relativamente pocos anilladores y estudios usando técnicas de anillado en el Perú. Tampoco hay peruanos certificados en anillamiento de aves rapaces, colibríes y anátidos, una situación que limita la posibilidad de un eventual proceso de certificación en estos grupos taxonómicos (Tabla 1).

\section{¿Cómo iniciarse en el anillamiento de aves en} el Perú?

El PAC ha tomado la iniciativa de promover la creación de estaciones de anillado de esfuerzo constante en diversos departamentos del país (Ica, Iquitos, Lima, Madre de Dios y Piura), los cuales junto a otras instituciones privadas tales como la Red de Anillamiento de Aves de Arequipa (RAAA), la Asociación Fauna Forever (FF) y la Reserva Ecológica Taricaya (RET), promueven el anillamiento científico bajo los estándares NABC. Cabe resaltar además que esta última institución alberga la primera estación de anillado de esfuerzo constante fundada en el país, operando ininterrumpidamente desde el 2005. Un objetivo de estas estaciones es de capacitar futuros anilladores interesados en el estudio de aves neotropicales y prepararlos para futuras sesiones de certificación.

¿Cómo ha sido posible la realización de estas sesiones de certificación en el Perú?

Entre el 2017 y 2018, CORBIDI, a través de sus estaciones (la Estación Biológica Río Santa Eulalia y el Observatorio de Aves de Loreto) y proyectos de anillado (Proyecto de Anillamiento de Aves Playeras), ha organizado las dos primeras sesiones de certificación NABC con mención en aves paserinas y la primera sesión de certificación con mención en aves playeras en el país bajo el auspicio de la Asociación de Ornitólogos de Campo (AFO), el WHSG, y el NABC. Estos eventos estuvieron dirigidos a investigadores provenientes de diversos países de América Latina y otras regiones del mundo intere-

Tabla 1. Número de profesionales peruanos certificados por el Consejo Norteamericano de Anillado en sus distintas menciones y niveles hasta finales del 2018.

\begin{tabular}{cccccc}
\hline Mención & $\begin{array}{c}\text { Paseriformes } \mathbf{y} \\
\text { Cuasi-paseriformes }\end{array}$ & Playeras & Rapaces & Colibríes & Anátidos \\
\hline Asistente & 0 & 1 & 0 & 0 & 0 \\
Anillador & 8 & 0 & 0 & 0 & 0 \\
Instructor & 5 & 1 & 0 & 0 & 0 \\
\hline
\end{tabular}


sados en el estudio de aves neotropicales y aves playeras migratorias. Como resultado de estos eventos de certificación, el número total de profesionales peruanos certificados, hasta finales del 2018, asciende a 15 (Tabla 1).

\section{¿En qué consisten estos procesos de certifica-} ción realizados recientemente en Perú?

Para el caso de la certificación con mención en aves paseriformes y cuasi-paseriformes, el candidato deberá optar primero por la certificación a nivel de Anillador, a través de la rendición de un examen teórico escrito, un examen de especímenes y una serie de exámenes prácticos de experiencia en campo (manejo de estaciones de anillado, extracción, manipulación de aves, manejo de datos y uso de redes de niebla) (NABC 2018). Una vez alcanzado el nivel de Anillador, además de contar con un puntaje mayor o igual que el $90 \%$ del examen escrito, una excelente capacidad de entrenar nuevos anilladores con diferentes niveles de experticio, y la capacidad de evaluar las competencias y habilidades de otros candidatos para el nivel de Anillador, el candidato podrá optar por la certificación a nivel de Instructor; proceso que contempla la rendición de un examen práctico de experiencia en campo, una entrevista personal, y presentaciones orales que evalúan las competencias y habilidades del candidato descritas previamente (NABC 2018).

Para el caso de la certificación con mención en aves playeras, el proceso es distinto y requiere de la participación en un taller de entrenamiento y certificación, rendición y aprobación de un examen escrito, y la compleción de un formulario de verificación de habilidades requeridas para anillar el grupo de aves en mención firmado por el candidato y sus instructores calificados (Gratto-Trevor 2018). Una vez completada todas las etapas, todos los documentos concernientes a este proceso son compilados y remitidos al Comité de Certificación del NABC, el cual posteriormente emitirá una decisión final sobre el nivel alcanzado por el candidato (Gratto-Trevor 2018). La certificación con mención en anátidos es similar al proceso con mención en aves playeras, mientras que la certificación en aves rapaces es un proceso híbrido que requiere de la participación en un taller de entrenamiento y una serie de exámenes prácticos de experiencia en campo. El proceso de certificación con mención en colibríes se encuentra actualmente en desarrollo. Es importante resaltar que todos los procesos de certificación mencionados anteriormente requieren de la revisión de material bibliográfico previo al desarrollo del taller de entrenamiento y/o sesión de certificación (NABC 2018).

\section{Futuros pasos}

Finalmente, el PAC busca implementar un programa certificación anual bajo los estándares de la NABC con el fin de incrementar el número de estaciones de investigación de la biología de las aves que usen el anillamiento como herramienta, así como del número de anilladores trabajando bajo un estándar consolidado. Además, el PAC busca alcanzar una representatividad que nos permita realizar estudios comparativos a lo largo de las diferentes regiones biogeográficas de país, fortaleciendo así el programa de anillamiento de aves en el Perú.

\section{Literatura citada}

Alza L., E. Bautista, M. Smith, N. Gutierrez-Pinto, A. Astie, \& K.G. McCracken. 2017. Capture efficiency of torrent ducks by the active mist-net method. Wildlife Society Bulletin 41:370-375. https://doi.org/10.1002/wsb.757

Antezana M. 2019. Patrones de muda y determinación de la edad del Mosquerito Silbador (Camptostoma obsoletum) (Temmick, 1824). Tesis de Licenciatura, Universidad Nacional Agraria La Molina, Lima, Perú, pp. 1-110.

Baillie S., F. Bairlein, J. Clark, C. du Feu, W. Fiedler, T. Fransson, J. Hegelbach, R. Julliard, Z. Karcza, L.F. Keller, M. Kestenholz, M. Schaub, \& F. Spina. 2007. Bird Ringing for Science and Conservation. EURING, Thetford, United Kingdom, pp. 1-33.

Baillie S. R., R.A. Robinson, J.A. Clark, \& C.P. Redfern. 2009. From individuals to flyways: the future of marking birds for conservation. Ringing \& Migration 24(3): 155-161. https://doi.org/10.1080/03078698.2009.9674386

Beingolea 0. 2009. Is illegal take affecting the Peregrine Falcon population in central Peru: why and how? In Tundra to tropics: connecting birds, habitats and people (Rich T. D., C. Arizmendi, D.W. Demarest, and C. Thompson. Eds.). Proceedings of the Fourth International Partners in Flight Conference, 13-16 February 2008, Partners in Flight, McAllen, Texas, pp.171-178.

Buckley P.A., C.M. Francis, P. Blancher, D.F. DeSante, C.S. Robbins, G. Smith, \& P. Cannell. 1998. The North American Bird Banding Program: Into the 21st Century. Journal of Field Ornithology, 69:511-529.

Calvo Mac C.L.G. 2016. Indicadores morfométricos de condiciones ambientales en un ave marina: el zarcillo (Larosterna inca). Tesis de Magíster Scientiae, Universidad Peruana Cayetano Heredia, Lima, Perú, pp. 1-62. http://repositorio.upch.edu.pe/handle/upch/326

Chumpitaz-Trujillo K.M. 2018. Estrategia y ciclo de muda de Catamenia analis (Aves: Thraupidae) y criterios para la determinación de su edad y sexo. Tesis de Licenciatura, Universidad Nacional Agraria La Molina, Lima, Perú, pp. 1-101.

Chumpitaz-Trujillo K.M., Newell, F.L., \& Ausprey, I.J. 2018. Utilización de patrones de muda para determinar la edad de la Reinita de corona rojiza (Myiothlypis coronata). Ornitología Neotropical 29(2): 75-81.

Díaz A. 2015. Patrones de muda y criterios de determinación de edad y sexo para Pipraeidea bonariensis. Tesis de Licenciatura, Universidad Nacional Federico Villarreal, Lima, Perú, pp. 1-82.

Díaz A., E. Berrocal, J. Salvador, F. Hernández, K. Chumpitaz, Y. Tenorio, \& T. Poma. 2015. Nuevos registros y actualización de la distribución de la Candelita Americana (Setophaga ruticilla) en el Perú. Boletín de la Unión de Ornitólogos del Perú 10(1):17-27.

Díaz A. \& G. Dimming. 2017. Distribution of Violaceous QuailDove Geotrygon violacea in Peru with four new localities. Cotinga 39:40-43.

Díaz O.A., F. Hernández, L. Alza, K. Chumpitaz, J. Salvador, E. Berrocal, Y. Tenorio, T. Poma, C. Santos \& J. Iannacone. 2018. Age determination based on molt patterns and skull ossification in the Blue-and-yellow tanager ( $\mathrm{Pi}$ praeidea bonariensis). Ornitología Neotropical 29(2): 51-61.. 
Doscher M. 2005. Bander Certification - Why? North American Bird Bander 30(3): 115.

Franke I. 2012. Uso de anillos para conocer las aves Peruanas. Disponible en http://avesecologaymedioambiente. blogspot.com/2012/02/uso-de-anillos-para-conocer-las-aves.html [Accedido 21 Agosto 2018].

Fuentes H. 1971. Resultados de los anillamientos en alcatraz (Pelecanus occidentalis thagus). Tesis de Bachiller, Universidad Nacional de San Agustín, Arequipa, Perú, pp. 1-37.

Gonzáles 0. 2004. Ecología de aves en un parque urbano de la ciudad de Lima. Tesis de Magíster Scientiae, Universidad Nacional Mayor de San Marcos, Lima, Perú, pp. 1-80.

Gonzáles O. \& E. Málaga. 1997. Estudios preliminares de la ecología de Xenospingus concolor en el Perú. In Armonía/ BirdLife Actas del III Encuentro Boliviano para la Conservación de las Aves, Santa Cruz, Bolivia.

Gonzáles O. \& M. La Torre-Cuadros. 2001. Análisis de hábitat del fringilo apizarrado (Xenospingus concolor) en la costa sur del Perú. Ornitología Neotropical, 12(2):153163.

Gratto-Trevor C.L. 2018. The North American Bander's Manual for Shorebirds. North American Banding Council Publication Committee, Saskatchewan, Canada. pp1-65.

Guallar S. 2015. El anillamiento: el método justifica los fines. Revista de anillamiento, 34:40-47.

Guevara-Torres D.R., J. Salvador, M. Antezana, F. Hernández, K. Chumpitaz, \& P. Saravia. 2017. Registros de Vireo flavoviridis en la costa central del Perú. Boletín de la Unión de Ornitólogos del Perú 12(2):15-19.

Herman C.M. 1944. The blood protozoa of North American birds. Bird-banding 15(3):89-112.

Jordán S., \& D. Cabrera. 1960. Algunos resultados de las anillaciones de Guanay (Phalacrocorax bougainvillii) efectuadas durante 1939-1941 y 1949-1953. Boletín de la Compañía Administradora del Guano 36(1):11-26.

Kennedy J.J., J. Heavyside, J.E. Jankowski, \& M.N. Scholer. 2018. Molt patterns and sexing and aging criteria for ten species of high elevation landbirds from southeastern Peru. Ornitología Neotropical, 29(2):63-73.

Koepcke M. 1959. Aves marcadas con anillos. Pesca y Caza [Ministerio de Agricultura, Dirección de Pesquería y Caza, Lima], 9: 73-75.

Mandujano-Collantes J.R. 2016. Patrones de muda de Zonotrichia capensis (Muller, 1776) (Aves: Emberezidae) en el bosque montano tropical de la provincia de Huarochirí - departamento de Lima. Tesis de Bachiller. Universidad Nacional de la Amazonia Peruana, Loreto, Perú, pp. 1-73.

Moreno M.I., P. Elizondo \& C.J. Ralph. 2011. Proceedings of the fourth WHBBN workshop, Brazil 2010. Western Hemisphere Bird Banding Network.

Myers J. P., M.A. Sallaberry., E. Ortiz., G. Castro, L. Gordon, J. L. Maron, C.T. Schick, E. Tablio, P. Antas, \& T. Bellow. 1990. Migration routes of new world sanderlings (Calidris alba). The Auk 107(1):172-180.

Nolazco S., \& J. Roper. 2014. The challenge of estimating population trends in the endangered Peruvian Plantcutter (Phytotoma raimondii) and implications for conservation. Ornitologia Neotropical 25(2):125-134.

North American Banding Council. 2003a. Guía de estudio del anillador de norteamérica. North American Banding Council Publication Committee, California, USA, pp1-78.
North American Banding Council. 2003b. Guía de entrenamiento del instructor de anilladores de aves paseriformes de Norteamérica. The North American banders' study guide. North American Banding Council Publication Committee, California, USA, pp1-32.

North American Banding Council (eds). 2018. Taxonomic Working Groups: Passerines and Near-passerines. Disponible en: https://www.nabanding.net/passerinesand-near-passerines/ [Accedido 21 Agosto 2018].

Plenge M.A. 2018. Referencias bibliográficas de las aves del Perú. Lima, Perú. Boletín de la Unión de Ornitólogos del Perú. Disponible en: https://drive.google.com/ file/d/1CBHV5wHEbxRkqcF4uDKcYP2REXo6gX7R/ view

Pinilla J. 2000. Manual para el anillamiento científico de aves. SEO/BirdLife y Dirección General de Conservación de la Naturaleza-Ministerio de Medio Ambiente, Madrid, España, pp. 1-160.

Pulido V., J. Jahncke, P. Makamatsu, \& C. Flores. 1996. Conservation of Charadriiformes on the Peruvian coast. International Wader Studies 8:55-61.

Ralph, C. J., G.R. Geupel, P. Pyle, T.E. Martin, D.F. DeSante, D. F., \& B. Milá. 1996. Manual de métodos de campo para el monitoreo de aves terrestres. General Technician Report: PSW-GTR-159. Pacific Southwest Research Station, Forest Service, U.S. Department of Agriculture. California, USA, pp.1-51.

Remsen Jr., J.V., C.D. Cadena, A. Jaramillo, M. Nores, J.F. Pacheco, J. Pérez-Emán, M.B. Robbins, F.G. Stiles, D.F. Stotz \& K.J. Zimmer. 2018. A classification of the bird species of South America. American Ornithologists' Union. <http://www.museum.lsu.edu/ Remsen/SACCBaseline.htm> Fecha de consulta: el 30/03/2018.

Sarango J. 1992. Anillamiento del Playero Blanco en la Reserva Nacional de Paracas. Volante Migratorio, 18:15-16.

Scholer M.N., C.L. Merkord, G.A. Londoño, \& J.E. Jankowski. 2018. Minimum longevity estimates for some Neotropical landbirds of southeastern Peru. The Wilson Journal of Ornithology. 130 (3):818-823. https://doi. org/10.1676/17-095.1

Stotz D. F., J.W. Fitzpatrick, T. A. Parker III, \& D. K. Moskovits.1996. Neotropical birds: ecology and conservation. University of Chicago Press.

Tautin J. 2005. One hundred years of bird banding in North America. In: C.J. Ralph; T.D. Rich (Ed.). Bird Conservation Implementation and Integration in the Americas: Proceedings of the Third International Partners in Flight Conference. 2002 March 20-24; Asilomar, California, Volume 1. USDA Forest Service General Technical Report PSW-GTR, 191: 815-816.

Tavera E.A., D.B. Lank, and P.M. González, P. M. 2016. Effects of migration distance on life history strategies of Western and Semipalmated sandpipers in Perú. Journal of Field Ornithology 87:293-308. https://doi. org/10.1111/jofo.12164.

Torres-Vásquez M.I., J. Ungvari-Martin, \& M. Salo. 2018. Muda y reproducción en aves de sotobosque de la Reserva Nacional Allpahuayo Mishana, Loreto, Perú. Ornitología Neotropical 29(1):229-239

Ungvari-Martin J., C.M. Heckscher, \& K.A. Hobson. 2016. Interannual site fidelity and breeding origins of Gray-cheeked Thrushes in white sand forests of the Peruvian Amazon. Journal of Field Ornithology 87(1):55-64. https://doi.org/10.1111/jofo.12133 
Villarán A. 2002. El anillamiento: un método de plena vigencia para el estudio científico de las aves. Ecología 16:433449.

Villavisencio A. 1990. Anillamiento de aves playeras en la Reserva Nacional de Paracas (Perú). Volante Migratorio, 15: 22-23.

Vogt W. 1942. Informe sobre las aves guaneras. Boletín de la Compañía Administradora del Guano, 18(3):1-132.

Zavalaga C.B. \& R. Paredes. 1997. Humboldt Penguins at Punta San Juan, Peru. Penguin Conservation 10(1):6-8.

\section{Agradecimientos:}

Agradecemos a Alfredo Begazo, Fernando Angulo, Luis Alza, Andrea Paterson, y C. John (CJ) Ralph por sus valiosos comentarios y observaciones sobre el artículo.

Conflicto de intereses:

Los autores no incurren en conflictos de intereses.

Rol de los autores:

AD, FH, MU, JS, ET, DG, RK, TV redactaron y aprobaron el manuscrito.

Fuentes de financiamiento:

El presente trabajo no tuvo un organismo financiador.

Aspectos éticos / legales:

El presente trabajo no incurre en problemas éticos o legales 